\title{
A New Simultaneous Identification of the Harmonic Excitations and Nonlinear Damping of Forced Damped Nonlinear Oscillations: A Parametric Approach
}

\author{
T. S. Jang \\ Department of Naval Architecture and Ocean Engineering, Pusan National University, Busan 609-735, Republic of Korea \\ Correspondence should be addressed to T. S. Jang; taek@pusan.ac.kr
}

Received 27 September 2012; Revised 21 December 2012; Accepted 23 December 2012

Academic Editor: George Jaiani

Copyright (C) 2013 T. S. Jang. This is an open access article distributed under the Creative Commons Attribution License, which permits unrestricted use, distribution, and reproduction in any medium, provided the original work is properly cited.

\begin{abstract}
This paper presents a novel, general method that is aimed at identifying both the harmonic excitations and nonlinear damping of forced nonlinear oscillation systems. An inverse formalism is developed for the identification method, which is shown to be well posed. That is, the inverse formalism has a unique solution and it depends continuously on the data. The unique solution is derived in the closed form. In addition to the mathematical analysis, a numerical example involving a highly nonlinear system is examined for demonstrating the workability of the proposed method for simultaneously identifying both the two aforementioned excitations and nonlinear damping.
\end{abstract}

\section{Introduction}

Over the past decades, a considerable number of studies have been made on forced oscillations arising in many diverse fields of science and engineering. This is mainly because the studies are closely related in the investigation of system performance such as the effective control and the estimation of the structure lifetime. Thus, as a forward problem, many researchers investigated nonlinear responses and stability analysis of the second-order nonlinear differential equations of a general form which have a periodic forcing term. However, these studies strongly depend on how accurately we can estimate the characteristics of forced loading condition or/and nonlinear damping. This is regarded as an inverse problem of the system identification, which is not only essential but crucial for the forward problem.

There are a variety of researches regarding the system identification devoted to the study about damping [1]. For example, Iourtchenko and Dimentberg [2] suggested a procedure for in-service identification of the damping based on the stochastic averaging method from a measured stationary response. Jang et al. [3, 4] proposed a new nonparametric method to recover the functional form of both nonlinear damping and nonlinear restoring forces of the dynamic system using the transient data. As for the force identification, there are also a lot of articles [5]. Doyle [6] studied a waveletbased deconvolution method to recover impact forces using experimentally measured responses. Zen et al. [7] proposed a method to obtain contact forces from experimental measurement of a single impact. Jang et al. [8] introduced a new technique for the identification of external loading of long time duration, which is nonharmonic but periodic, acting on a nonlinear dynamic system.

As mentioned above, although a large number of studies have been carried out on the system identification of nonlinear damping or external force, little is known about their combinations, that is, the simultaneous identification of nonlinear damping as well as external force. In fact, many researchers in engineering fields have wondered if the problem of the simultaneous identification is mathematically solvable. Motivated by this, an inverse formalism is, in this present work, mathematically developed for identifying both the nonlinear damping and external forces in a forced nonlinear system.

In this study, we propose a method for the simultaneous identification, which is a new procedure with remarkable 
improvements. First, based on the concept of a zero-crossing time, we develop an inverse formalism which is linear, even if the original dynamical equation of motion is nonlinear. Second, we prove that the inverse formalism has a unique solution and its closed form solution is derived, which makes the proposed method quite simple and straightforward. Third, the inverse formalism developed is shown to have stability properties of solution. This means that the method by the inverse formalism work without the use of any regularization, so that it can have a wide range of physical applications. This contrasts the usual inverse problems illposed in the sense of stability $[3,4,8-10]$, which require an additional process of regularization.

Finally, given the model nonlinear differential equation concerning the quadratic nonlinear damping and cubic nonlinear restoring characteristics, the proposed method's workability for simultaneously identifying both excitations and nonlinear damping is demonstrated through a numerical example.

\section{Forced Damped Nonlinear Oscillation}

We consider a nonlinear damped dynamical system, subject to an external harmonic load $\Gamma \cdot \cos \omega t$, of an amplitude $\Gamma>0$ and frequency $\omega>0$. The equation of motion is a second-order nonlinear ordinary differential equation for the response $q$

$$
m \ddot{q}+D(\dot{q})+R(q)=\Gamma \cdot \cos \omega t, \quad t>0 .
$$

Here, the constant $m>0$ represents the mass of a particle of an oscillator. The term $R(q)$ is a general nonlinear restoring force and the term $D(\dot{q})$, appearing in (1), is the nonlinear damping of a system, which is assumed to have a quadratic nonlinear term

$$
D(\dot{q})=c_{1} \dot{q}+c_{2} \dot{q}|\dot{q}|
$$

where $c_{1}$ and $c_{2}$ are the linear and nonlinear damping coefficients, respectively. The functional form, (2), of nonlinearity is often encountered in the area of engineering field [11].

The sum of the inertia force $m \ddot{q}$ and restoring force $R(q)$ and the derivative of the sum will play an important role in identifying both the excitation $\Gamma$ and nonlinear damping $D(\dot{q})$ in (1). We define the sum as

$$
J(q, \ddot{q}) \equiv m \ddot{q}+R(q) .
$$

The derivative of $J$ with respect to time then becomes

$$
\begin{aligned}
\dot{J} & =\frac{\partial J}{\partial q}(q, \ddot{q}) \cdot \dot{q}+\frac{\partial J}{\partial \ddot{q}}(q, \ddot{q}) \cdot \ddot{q} \\
& =\frac{d R}{d q}(q) \cdot \dot{q}+m \ddot{q} .
\end{aligned}
$$

Noting that (1) is equivalent to

$$
J=\Gamma \cdot \cos \omega t-D(\dot{q})
$$

we can express $\dot{J}$ in terms of the excitation and damping as follows:

$$
\dot{J}=-\Gamma \omega \cdot \sin \omega t-\frac{d D}{d \dot{q}} \ddot{q}
$$

by differentiating (5) with respect to time.

In what follows, we assume that (1) has two dynamic responses of $T(=2 \pi / \omega)$-periodic limit cycle solutions $q_{i}(t)$, which are forced by $\Gamma_{i}$ for $i=1,2$

$$
m \ddot{q}_{i}+D\left(\dot{q}_{i}\right)+R\left(q_{i}\right)=\Gamma_{i} \cdot \cos \omega t, \quad t>0 \text { for } i=1,2 .
$$

The response analysis of the nonlinear damped dynamical system considered can be regarded as a forward problem, whose solution procedures are well established theoretically as well as numerically. However, an inverse problem can now be formulated in the form of a question: given a motion response of the nonlinear dynamical system, is it possible to identify the excitations and nonlinear damping of the system at the same time? The question may be interesting mathematically as well as practically. In order to answer the question, we will begin with introducing a zero-crossing time in the next section.

\section{Some Lemmas on Zero-Crossing Time}

This section is concerned with a zero-crossing time associated with motion responses of a system. Thereby, we derive some lemmas which are essential in the present study. We start by defining a zero-crossing time in terms of $J$ for $i=1,2$, denoted by $\left(t_{J}^{\mathrm{ze}}\right)_{i}$, satisfying

$$
J_{i}\left[\left(t_{J}^{\mathrm{ze}}\right)_{i}\right]=0 \text { for } i=1,2 .
$$

Here, $J_{i}$ stands for the sum (3) which corresponds to $q_{i}$, that is, a function from $(0, \infty)$ to $\mathbb{R}$

$$
J_{i} \equiv m \ddot{q}_{i}+R\left(q_{i}\right) .
$$

Physically, the zero-crossing time defined in (8) is a useful concept for finding the force balancing between the excitation and the damping in a forced damped oscillatory motion of (7) as discussed in the following lemma.

Lemma 1. Suppose that $\left(t_{J}^{z e}\right)_{i}, i=1,2$, is a zero-crossing time in terms of $J$ for the system (7). Then the following holds:

$$
\Gamma_{i} \cdot \cos \left[\omega\left(t_{J}^{z e}\right)_{i}\right]=D\left\{\dot{q}_{i}\left[\left(t_{J}^{z e}\right)_{i}\right]\right\} \quad \text { for } i=1,2 .
$$

Proof. A simple substitution of (9) into (7) yields

$$
J_{i}=\Gamma_{i} \cdot \cos \omega t-D\left(\dot{q}_{i}\right) .
$$

Since (11) remains valid for $0<t<\infty$, it should also hold for the zero-crossing time of $\left(t_{J}^{\mathrm{ze}}\right)_{i}>0$. However, the left hand side of (11) vanishes for the zero-crossing time $\left(t_{J}^{\mathrm{ze}}\right)_{i}$ due to the definition (8). That is,

$$
0=\Gamma_{i} \cdot \cos \left[\omega\left(t_{J}^{\mathrm{ze}}\right)_{i}\right]-D\left\{\dot{q}_{i}\left[\left(t_{J}^{\mathrm{ze}}\right)_{i}\right]\right\} \quad \text { for } i=1,2
$$

which immediately proves (10). 
Next, we introduce a zero-crossing time in terms of acceleration, $\left(t_{\mathrm{acc}}^{\mathrm{ze}}\right)_{i}$ for $i=1,2$, such that

$$
\ddot{q}_{i}\left[\left(t_{\mathrm{acc}}^{\mathrm{ze}}\right)_{i}\right]=0 \text { for } i=1,2,
$$

where $\ddot{q}_{i}$ denotes an acceleration response of (7). We notice that the time rate of change of the damping is always zero at a zero-crossing time in terms of acceleration, which shall be examined in Lemma 2.

Lemma 2. For the function $\dot{J}_{i}$ defined by

$$
\dot{j}_{i}(t)=\frac{d J}{d t}\left[q_{i}(t), \ddot{q}_{i}(t)\right], \quad \text { for } i=1,2,
$$

one has

$$
\dot{J}_{i}\left[\left(t_{\text {acc }}^{z e}\right)_{i}\right]=-\Gamma_{i} \omega \cdot \sin \left[\omega\left(t_{\text {acc }}^{z e}\right)_{i}\right] \text { for } i=1,2 .
$$

Here $\left(t_{\text {acc }}^{z e}\right)_{i}$ refers to a zero-crossing time in terms of acceleration.

Proof. In the same way as Lemma 1, (14) should hold for the zero-crossing times in terms of acceleration, that is, $t=$ $\left(t_{\mathrm{acc}}^{\mathrm{ze}}\right)_{i}$, for $i=1,2$. However, (14) is rewritten as

$$
\dot{J}_{i}=-\Gamma_{i} \omega \cdot \sin \omega t-\frac{d D}{d \dot{q}_{i}} \ddot{q}_{i}
$$

in which the second term of the right hand side becomes zero because the acceleration vanishes when $t=\left(t_{\mathrm{acc}}^{\mathrm{ze}}\right)_{i}$ by the definition (13). It follows that the above equation becomes (15), which completes the proof.

\section{Inverse Formalism}

It is a high time that we develop an inverse formalism for the inverse problem raised by Section 2, based on the lemmas in the previous section. We recall that the excitations and nonlinear damping are parameterized by $\Gamma_{1}, \Gamma_{2}, c_{1}$, and $c_{2}$ in Section 2. This results in that the developed inverse formalism also involves the parameters of $\Gamma_{1}, \Gamma_{2}, c_{1}$, and $c_{2}$ as illustrated in Lemma 3.

Lemma 3 (inverse formalism). Equations (10) and (15) in Lemmas 1 and 2 constitute simultaneous linear algebraic equations for the four unknowns $\Gamma_{1}, \Gamma_{2}, c_{1}$, and $c_{2}$. These can be written in a compact way as follows:

$$
\mathbf{A x}=\mathbf{b} .
$$

Here, $\mathbf{A}$ is an operator from $\mathbb{R}^{4}$ to $\mathbb{R}^{4}$ whose matrix is

$$
[\mathbf{A}] \equiv\left[\begin{array}{cc}
\cos \left[\omega\left(t_{J}^{z e}\right)_{1}\right] & 0 \\
0 & \cos \left[\omega\left(t_{J}^{z e}\right)_{2}\right] \\
-\omega \cdot \sin \left[\omega\left(t_{a c c}^{z e}\right)_{1}\right] & 0 \\
0 & -\omega \cdot \sin \left[\omega\left(t_{a c c}^{z e}\right)_{2}\right] \\
-\dot{q}\left[\left(t_{J}^{z e}\right)_{1}\right] & -\dot{q}\left[\left(t_{J}^{z e}\right)_{1}\right]\left|\dot{q}\left[\left(t_{J}^{z e}\right)_{1}\right]\right| \\
-\dot{q}\left[\left(t_{J}^{z e}\right)_{2}\right] & -\dot{q}\left[\left(t_{J}^{z e}\right)_{2}\right]\left|\dot{q}\left[\left(t_{J}^{z e}\right)_{2}\right]\right| \\
0 & 0 \\
0 & 0
\end{array}\right]
$$

and $\mathbf{x}, \mathbf{b} \in \mathbb{R}^{4}$ are represented as (one will call them a solution vector and data vector, resp., in this paper)

$$
[\mathbf{x}] \equiv\left[\begin{array}{c}
\Gamma_{1} \\
\Gamma_{2} \\
c_{1} \\
c_{2}
\end{array}\right], \quad[\mathbf{b}] \equiv\left[\begin{array}{c}
0 \\
0 \\
\dot{J}_{1}\left[\left(t_{a c c}^{z e}\right)_{1}\right] \\
\dot{j}_{2}\left[\left(t_{a c c}^{z e}\right)_{2}\right]
\end{array}\right] .
$$

Proof. Equations (10) and (15) in Lemmas 1 and 2 can be rewritten as the following:

$$
\begin{gathered}
\Gamma_{1} \cdot \cos \left[\omega\left(t_{J}^{\mathrm{ze}}\right)_{1}\right]-c_{1} \dot{q}\left[\left(t_{J}^{\mathrm{ze}}\right)_{1}\right]-c_{2} \dot{q}\left[\left(t_{J}^{\mathrm{ze}}\right)_{1}\right]\left|\dot{q}\left[\left(t_{J}^{\mathrm{ze}}\right)_{1}\right]\right|=0 \\
\Gamma_{2} \cdot \cos \left[\omega\left(t_{J}^{\mathrm{ze}}\right)_{2}\right]-c_{1} \dot{q}\left[\left(t_{J}^{\mathrm{ze}}\right)_{2}\right]-c_{2} \dot{q}\left[\left(t_{J}^{\mathrm{ze}}\right)_{2}\right]\left|\dot{q}\left[\left(t_{J}^{\mathrm{ze}}\right)_{2}\right]\right|=0 \\
-\Gamma_{1} \omega \cdot \sin \left[\omega\left(t_{\mathrm{acc}}^{\mathrm{ze}}\right)_{1}\right]=\dot{J}_{1}\left[\left(t_{\mathrm{acc}}^{\mathrm{ze}}\right)_{1}\right] \\
-\Gamma_{2} \omega \cdot \sin \left[\omega\left(t_{\mathrm{acc}}^{\mathrm{ze}}\right)_{2}\right]=\dot{J}_{2}\left[\left(t_{\mathrm{acc}}^{\mathrm{ze}}\right)_{2}\right]
\end{gathered}
$$

It is straightforward to show that the above four equations are equivalent to the operator equation (17) from the definition of the operator $\mathbf{A}$, the vectors $\mathbf{x}$ and $\mathbf{b}$ of (18) and (19). This completes the proof.

\section{Method}

In the previous section, we developed an inverse formalism (17), whose solution procedure is to be established in this section. The formal expression for the solution may be symbolically written as

$$
\mathbf{x}=\mathbf{A}^{-1} \mathbf{b}
$$

where the notation $\mathbf{A}^{-1}$ denotes the inverse operator for the linear operator $\mathbf{A}$ of (18). According to linear algebra, it is well known that the inverse operator is bounded (or continuous), because it is defined on finite dimensional spaces. Thus, the solution $\mathbf{x}$ in (24) depends continuously on the data vector b: that is, the solution $\mathbf{x}$ is stable. The construction of $\mathbf{x}$ is examined through the following theorems.

Theorem 4 (excitations). The excitations $\Gamma_{i}$ for $i=1,2$ in (7) are identified as an analytical form

$$
\Gamma_{i}=\frac{-\dot{J}_{i}\left[\left(t_{a c c}^{z e}\right)_{i}\right]}{\omega \sin \left[\omega\left(t_{a c c}^{z e}\right)_{i}\right]}
$$

provided that $\sin \left[\omega\left(t_{\text {acc }}^{z e}\right)_{i}\right] \neq 0$ for $i=1,2$.

Proof. It is easy to notice that the formula of (25) immediately follows from the relations of (22) and (23). We first divide both sides of $(22)$ by $-\omega \cdot \sin \left[\omega\left(t_{\text {acc }}^{\text {ze }}\right)_{1}\right]$ to obtain $\Gamma_{1}$ as follows:

$$
\Gamma_{1}=\frac{-\dot{J}_{1}\left[\left(t_{\mathrm{acc}}^{\mathrm{ze}}\right)_{1}\right]}{\omega \cdot \sin \left[\omega\left(t_{\mathrm{acc}}^{\mathrm{ze}}\right)_{1}\right]} .
$$


Similarly, we have

$$
\Gamma_{2}=\frac{-\dot{J}_{2}\left[\left(t_{\mathrm{acc}}^{\mathrm{ze}}\right)_{2}\right]}{\omega \cdot \sin \left[\omega\left(t_{\mathrm{acc}}^{\mathrm{ze}}\right)_{2}\right]}
$$

from (23). This completes the proof.

The formulas of the damping coefficients $c_{1}$ and $c_{2}$ in (2) can also be obtained in an essentially simple way, as to be examined in Theorem 5.

Theorem 5 (nonlinear damping). The linear damping coefficient $c_{1}$ and the nonlinear damping coefficient $c_{2}$ in (2) are determined as

$$
\begin{aligned}
c_{1}= & \frac{\dot{J}_{2}\left[\left(t_{a c c}^{z e}\right)_{2}\right] \cdot \cos \left[\omega\left(t_{J}^{z e}\right)_{2}\right] \cdot\left|\dot{q}\left[\left(t_{J}^{z e}\right)_{1}\right]\right|}{\omega \sin \left[\omega\left(t_{a c c}^{z e}\right)_{2}\right] \dot{q}\left[\left(t_{J}^{z e}\right)_{2}\right]\left\{\left|\dot{q}\left[\left(t_{J}^{z e}\right)_{2}\right]\right|-\left|\dot{q}\left[\left(t_{J}^{z e}\right)_{1}\right]\right|\right\}} \\
& -\frac{\dot{J}_{1}\left[\left(t_{a c c}^{z e}\right)_{1}\right] \cos \left[\omega\left(t_{J}^{z e}\right)_{1}\right] \cdot\left|\dot{q}\left[\left(t_{J}^{z e}\right)_{2}\right]\right|}{\omega \sin \left[\omega\left(t_{a c c}^{z e}\right)_{1}\right] \dot{q}\left[\left(t_{J}^{z e}\right)_{1}\right]\left\{\left|\dot{q}\left[\left(t_{J}^{z e}\right)_{2}\right]\right|-\left|\dot{q}\left[\left(t_{J}^{z e}\right)_{1}\right]\right|\right\}},
\end{aligned}
$$

$$
\begin{aligned}
c_{2}= & \frac{\dot{J}_{1}\left[\left(t_{a c c}^{z e}\right)_{1}\right] \cos \left[\omega\left(t_{J}^{z e}\right)_{1}\right]}{\omega \sin \left[\omega\left(t_{a c c}^{z e}\right)_{1}\right] \cdot \dot{q}\left[\left(t_{J}^{z e}\right)_{1}\right]\left\{\left|\dot{q}\left[\left(t_{J}^{z e}\right)_{2}\right]\right|-\left|\dot{q}\left[\left(t_{J}^{z e}\right)_{1}\right]\right|\right\}} \\
& -\frac{\dot{J}_{2}\left[\left(t_{a c c}^{z e}\right)_{2}\right] \cos \left[\omega\left(t_{J}^{z e}\right)_{2}\right]}{\omega \sin \left[\omega\left(t_{a c c}^{z e}\right)_{2}\right] \dot{q}\left[\left(t_{J}^{z e}\right)_{2}\right]\left\{\left|\dot{q}\left[\left(t_{J}^{z e}\right)_{2}\right]\right|-\left|\dot{q}\left[\left(t_{J}^{z e}\right)_{1}\right]\right|\right\}} .
\end{aligned}
$$

Proof. It suffices to multiply (20) and (21) by the corresponding factors, then subtract them from each other. As a result of taking (25) into account, we find $c_{1}$ of (28) and then $c_{2}$ of (29), which completes the proof.

Remark 6. Because of the stability properties of solution $\mathbf{x}$, our solution procedure does not concern any kind of regularization, which is necessary for the usual inverse problems arising in science and engineering.

As to the solvability and uniqueness condition, the operator $\mathbf{A}$ in (18) should satisfy det $\mathbf{A} \neq 0$, which is to be discussed in the following theorem.

Theorem 7 (uniqueness). Let one denote by $\Delta$,

$$
\begin{aligned}
\Delta= & \sin \left[\omega\left(t_{a c c}^{z e}\right)_{1}\right] \cdot \sin \left[\omega\left(t_{a c c}^{z e}\right)_{2}\right] \dot{q}\left[\left(t_{J}^{z e}\right)_{1}\right] \dot{q}\left[\left(t_{J}^{z e}\right)_{2}\right] \\
& \times\left\{\left|\dot{q}\left[\left(t_{J}^{z e}\right)_{2}\right]\right|-\left|\dot{q}\left[\left(t_{J}^{z e}\right)_{1}\right]\right|\right\} .
\end{aligned}
$$

Then, the excitations and nonlinear damping in (7) can be identified in a unique way whenever $\Delta \neq 0$.

Proof. It suffices to notice that the formulas (25), (28), and (29) are valid if and only if the corresponding denominators are different from zero. After writing these restrictions, we obtain a condition equivalent to $\Delta \neq 0$, which completes the proof.

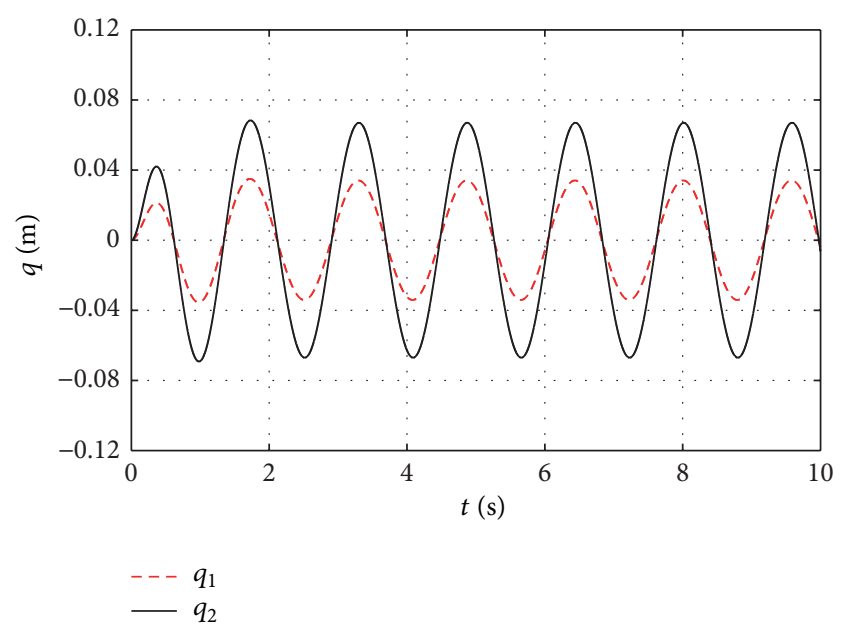

(a) Displacements: $q_{1}$ and $q_{2}$

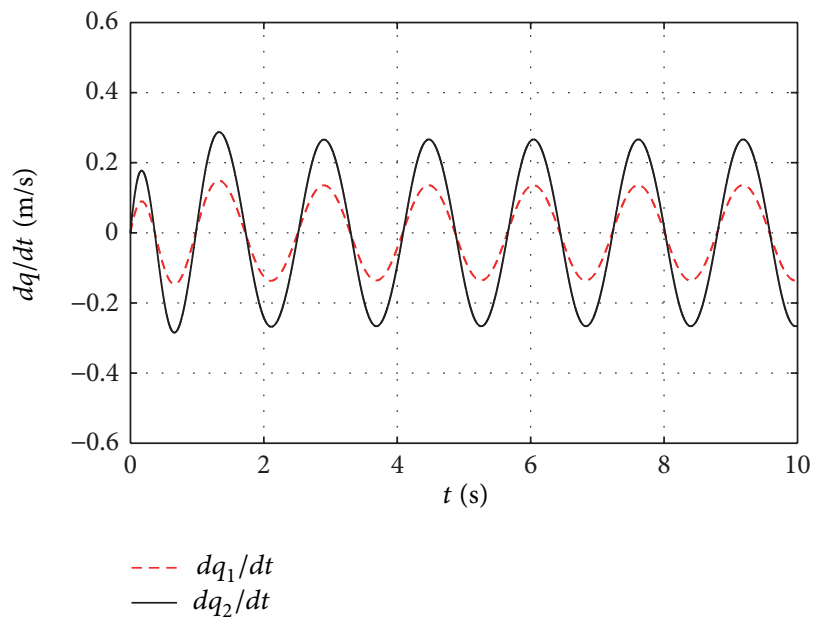

(b) Velocities: $\dot{q}_{1}$ and $\dot{q}_{2}$

Figure 1: System responses.

Remark 8. Theorem 7 tells us that the stable solution $\mathbf{x}$ is unique. This implies that the inverse formalism developed in the present study is a well-posed problem.

Our method for the simultaneous identification consists of the closed form solutions for the inverse formalism as in Theorems 4 and 5. However, the solutions are still expressed through the unknown zero crossing times. We thus are required to determine the unknown times for realizing the solutions, which is discussed in more detail in the next section.

\section{Numerical Example}

In this section, we will examine the workability of the proposed method to identify the excitations and nonlinear damping through numerical experiments. For that, let us assume that the restoring term $R(q)$ in (1) involves the cubic nonlinearity, that is,

$$
R(q)=k_{1} q+k_{2} q^{3}
$$




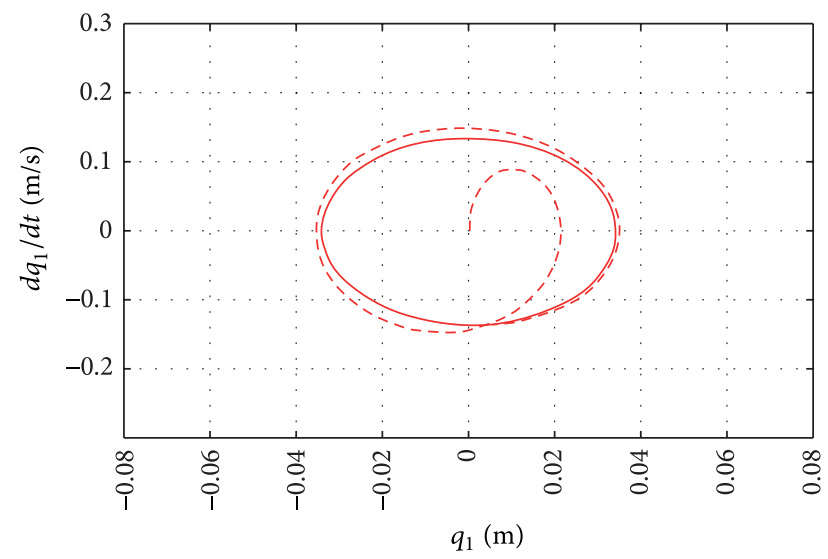

(a) $q_{1}$ and $\dot{q}_{1}$

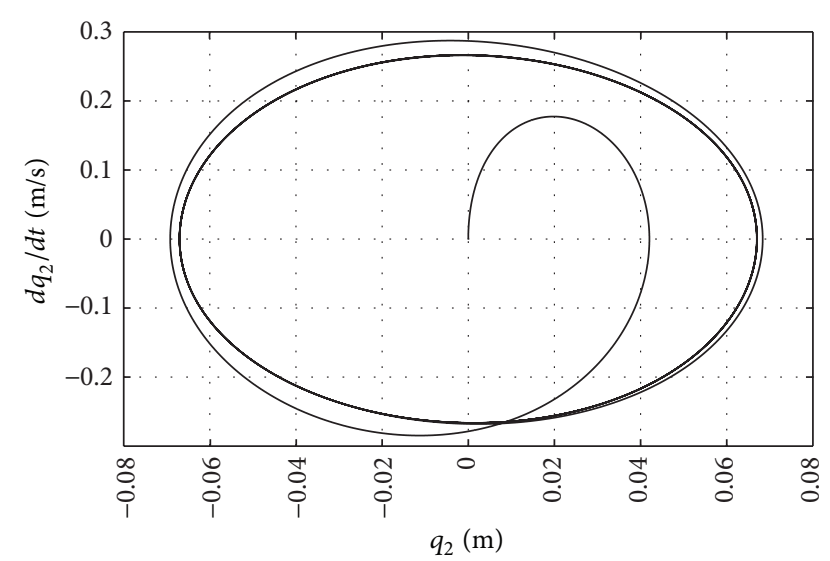

(b) $q_{2}$ and $\dot{q}_{2}$

FIgURE 2: Phase diagrams for the system responses.

where the values for $k_{1}$ and $k_{2}$ are chosen as 40 and 10 , respectively. Then, the responses for the system of (1) can be observed as following subsection.

6.1. System Responses. With the initial conditions imposed as

$$
q_{i}(0)=0, \quad \dot{q}_{i}(0)=0 \quad \text { for } i=1,2,
$$

we integrate (7) by employing Runge-Kutta schemes to seek the system responses of (7). Here, the mass is normalized as unit and the coefficients of $c_{1}$ and $c_{2}$ are set to be 4 and 2, respectively. The excitation frequency $\omega$ is taken as 4 with $\Gamma_{1}=$ 1 and $\Gamma_{2}=2$. The calculated responses of displacement and velocity of the system are depicted in Figure 1 and their phase diagrams in Figure 2.

6.2. Recovering Solutions by Zero-Crossing Time. As mentioned in Section 5, even if we derived the closed form solutions for the present identification, they depend on the unknown two zero-crossing times, namely, $\left(t_{J}^{\mathrm{ze}}\right)_{i}$ and $\left(t_{\mathrm{acc}}^{\mathrm{ze}}\right)_{i}$ in (8) and (13), respectively.

As a first case, we attempt to find the zero-crossing times from the steady-state region. By a proper numerical
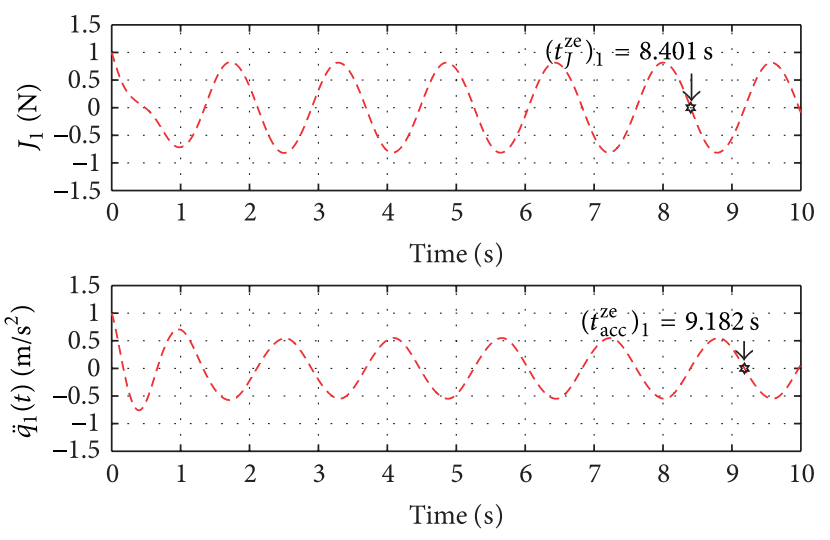

(a) $\left(t_{J}^{\mathrm{ze}}\right)_{1}$ and $\left(t_{\mathrm{acc}}^{\mathrm{ze}}\right)_{1}$
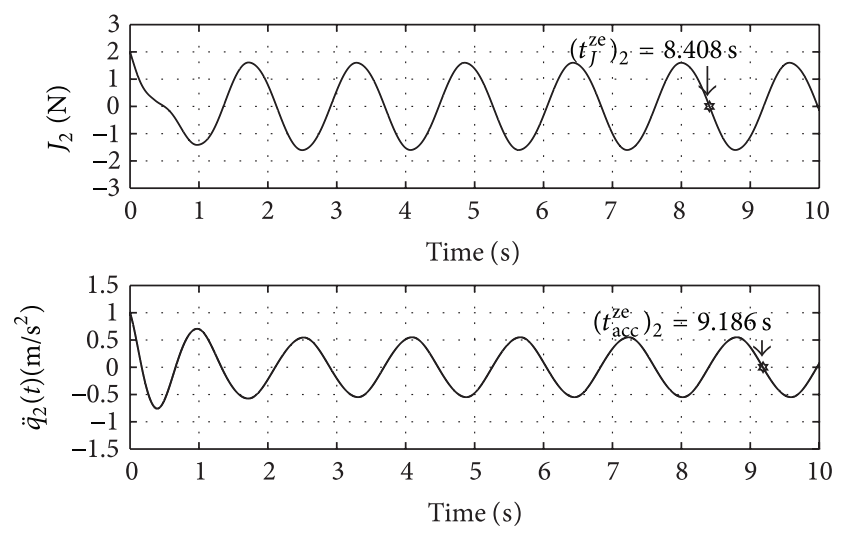

(b) $\left(t_{J}^{\mathrm{ze}}\right)_{2}$ and $\left(t_{\mathrm{acc}}^{\mathrm{ze}}\right)_{2}$

FIGURE 3: Illustration of zero-crossing times in the region of steady state.

TABLE 1: Identified coefficients compared with the exact ones: steady-state region.

\begin{tabular}{lcccc}
\hline & $c_{1}$ & $c_{2}$ & $\Gamma_{1}$ & $\Gamma_{2}$ \\
\hline Exact & 4 & 2 & 1 & 2 \\
Identified & 3.9761 & 2.0195 & 0.9988 & 2.0003 \\
Error (\%) & 0.6 & 1.0 & 0.1 & 0.02 \\
\hline
\end{tabular}

equation solver, the results are calculated as $\left(t_{J}^{\mathrm{ze}}\right)_{1}=8.401 \mathrm{sec}$, $\left(t_{J}^{\mathrm{ze}}\right)_{2}=8.408 \mathrm{sec},\left(t_{\mathrm{acc}}^{\mathrm{ze}}\right)_{1}=9.182 \mathrm{sec}$, and $\left(t_{\mathrm{acc}}^{\mathrm{ze}}\right)_{2}=9.186 \mathrm{sec}$, each of which is indicated in Figure 3 . We then substitute the zero-crossing times into (25), (28), and (29) for realizing the closed form solutions of identification. Table 1 presents the numerical values for the solutions by the proposed method, which is compared with the exact ones. In addition, timesimulated oscillatory motions using the identified excitations and damping by the proposed method are depicted in Figure 6, where the real (exact) time-simulated oscillatory motions also appear for comparison.

In a similar manner, as a second case, Figure 4 shows the zero-crossing times selected from the transient region, which are substituted again into (25), (28), and (29) for the identification. The results, compared with the exact ones, are 

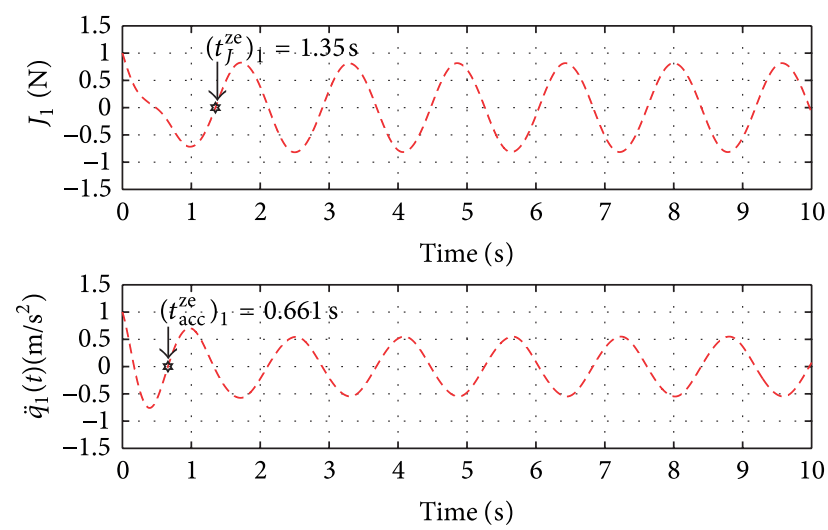

(a) $\left(t_{J}^{\mathrm{ze}}\right)_{1}$ and $\left(t_{\mathrm{acc}}^{\mathrm{ze}}\right)_{1}$
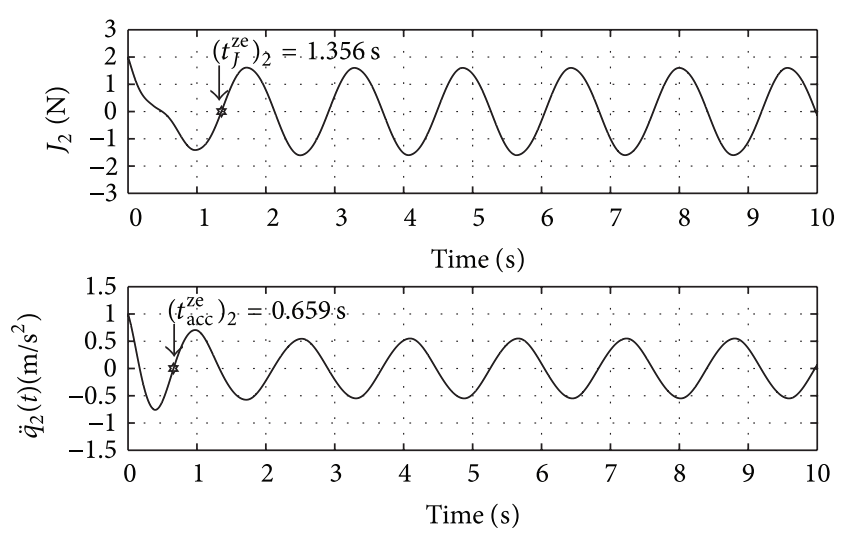

(b) $\left(t_{J}^{\mathrm{ze}}\right)_{2}$ and $\left(t_{\mathrm{acc}}^{\mathrm{ze}}\right)_{2}$

FIGURE 4: Illustration of zero-crossing times from the transient region.

TABLE 2: Identified coefficients compared with the exact ones: transient region.

\begin{tabular}{lcccc}
\hline & $c_{1}$ & $c_{2}$ & $\Gamma_{1}$ & $\Gamma_{2}$ \\
\hline Exact & 4 & 2 & 1 & 2 \\
Identified & 3.9120 & 2.4773 & 0.9984 & 2.0227 \\
Error $(\%)$ & 2.2 & 23.87 & 0.16 & 1.14 \\
\hline
\end{tabular}

listed in Table 2. Finally (third case), we choose $\left(t_{J}^{\mathrm{ze}}\right)_{i}$ from the transient region and $\left(t_{\mathrm{acc}}^{\mathrm{ze}}\right)_{i}$ from the steady-state region for each $i=1,2$ as shown in Figure 5. Their corresponding results are listed in Table 3 . For all three cases, the values for the condition numbers of $\mathbf{A}$ in (18) are of $O\left(10^{2}\right)$, meaning that our system equation (17) is well conditioned.

\section{Concluding Remarks}

As discussed in Section 1 of introduction, many of engineers have doubted if it is possible to identify not only the external forcing but also nonlinear damping characteristics by only using system responses. This is a basic idea underlying this study. We have introduced the concept of zero-crossings with
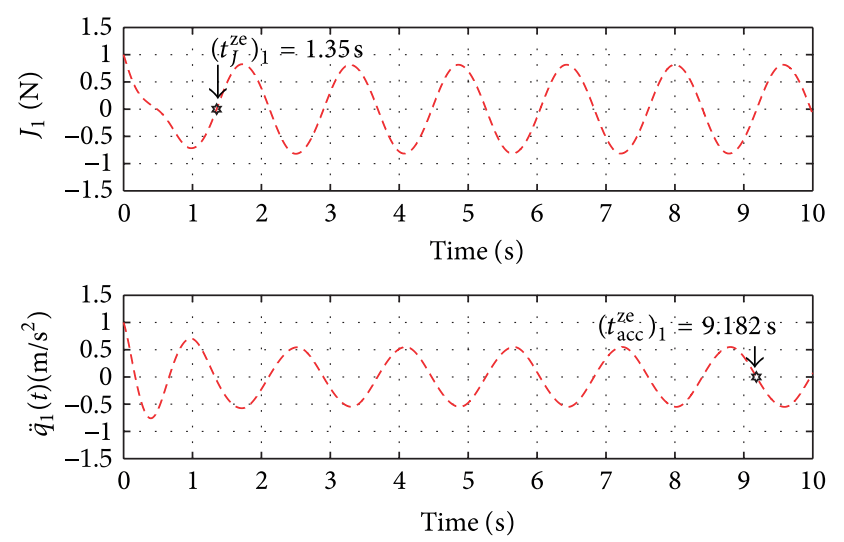

(a) $\left(t_{J}^{\mathrm{ze}}\right)_{1}$ and $\left(t_{\mathrm{acc}}^{\mathrm{ze}}\right)_{1}$
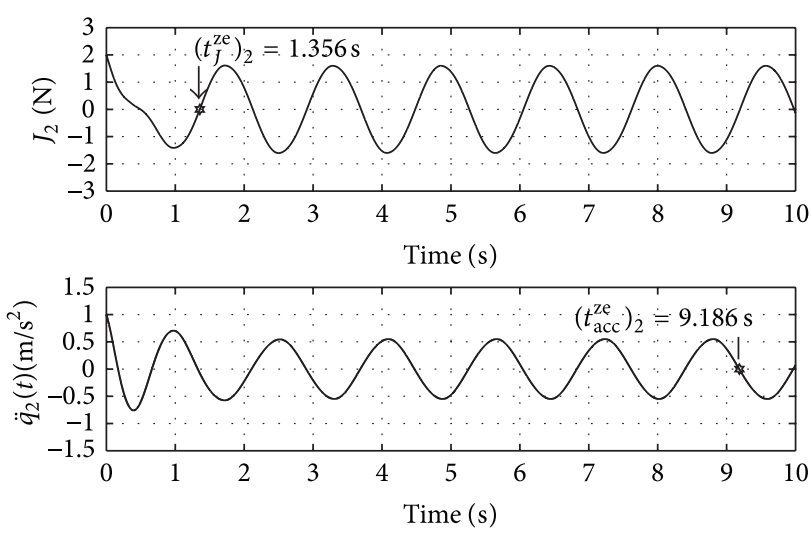

(b) $\left(t_{J}^{\mathrm{ze}}\right)_{2}$ and $\left(t_{\mathrm{acc}}^{\mathrm{ze}}\right)_{2}$

FIGURE 5: Illustration of zero-crossing times: $\left(t_{J}^{\text {ze }}\right)_{i}$ from the transient region and $\left(t_{\mathrm{acc}}^{\mathrm{ze}}\right)_{i}$ from the steady-state region $(i=1,2)$.

TABLE 3: Identified coefficients compared with the exact ones: $\left(t_{J}^{\mathrm{ze}}\right)_{i}$ - the transient region, $\left(t_{\mathrm{acc}}^{\mathrm{ze}}\right)_{i}$ - the steady-state region $(i=1,2)$.

\begin{tabular}{lcccc}
\hline & $c_{1}$ & $c_{2}$ & $\Gamma_{1}$ & $\Gamma_{2}$ \\
\hline Exact & 4 & 2 & 1 & 2 \\
Identified & 3.9702 & 2.0948 & 0.9988 & 2.0003 \\
Error (\%) & 0.75 & 4.74 & 0.1 & 0.02 \\
\hline
\end{tabular}

regard to the system responses, which becomes a crucial key to answer this question successfully. Thereby, we present a mathematical analysis for a systematic process of identification as well as a general method for the nonlinear simultaneous identification. Even though the numerical example provided herein is a highly nonlinear system, the proposed method yields a good approximation of the exact expression in identifying the two nonlinear physical quantities in a stable and accurate manner.

\section{Acknowledgments}

This research is supported by Basic Science Research Program through the National Research Foundation of Korea (NRF) 


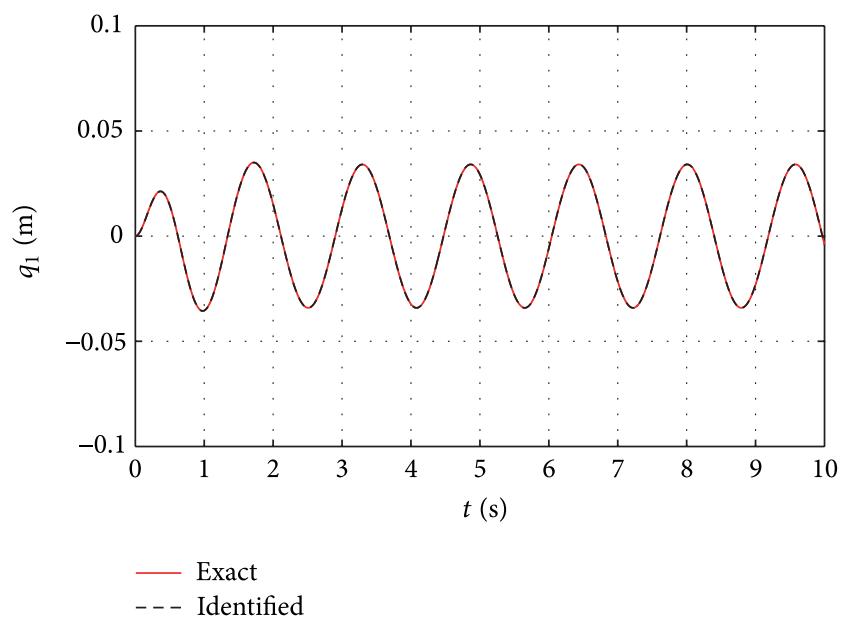

(a)

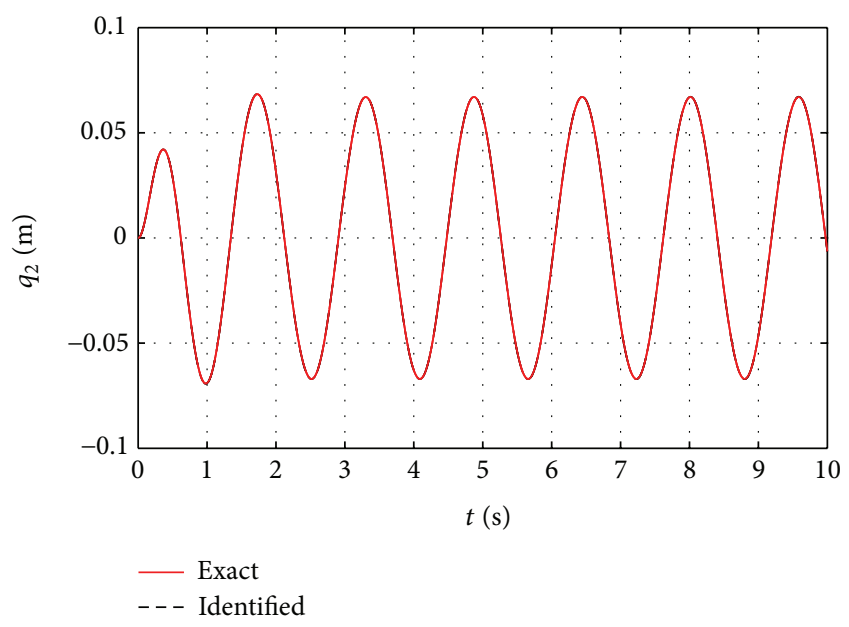

(b)
[3] T. S. Jang, H. S. Choi, and S. L. Han, "A new method for detecting non-linear damping and restoring forces in nonlinear oscillation systems from transient data," International Journal of Non-Linear Mechanics, vol. 44, pp. 801-808, 2009.

[4] T. S. Jang, "Non-parametric simultaneous identification of both the nonlinear damping and restoring characteristics of nonlinear systems whose dampings depend on velocity alone," Mechanical Systems and Signal Processing, vol. 25, pp. 1159-1173, 2011.

[5] S. F. Masri and T. K. Caughey, "A nonparametric identification technique for nonlinear dynamic problems," ASME Journal of Applied Mechanics, vol. 46, pp. 433-447, 1979.

[6] J. Doyle, "A wavelet deconvolution method for impact force identification," Experimental Mechanics, vol. 37, pp. 403-408, 1997.

[7] Q. H. Zen, M. Chapin, and D. B. Bogy, "A force identification method for slider/disk contact force measurement," IEEE Transactions on Magnetics, vol. 36, pp. 2667-2670, 2000.

[8] T. S. Jang, H. S. Baek, H. S. Choi, and S. G. Lee, "A new method for measuring nonharmonic periodic excitation forces in nonlinear damped systems," Mechanical Systems and Signal Processing, vol. 256, pp. 2219-2228, 2011.

[9] T. S. Jang, H. S. Back, S. L. Han, and T. Kinoshita, "Indirect measurement of the impulsive load to a nonlinear system from dynamic responses: inverse problem formulation," Mechanical Systems and Signal Processing, vol. 24, pp. 1665-1681, 2010.

[10] T. S. Jang, S. L. Han, and T. Kinoshita, "An inverse measurement of the sudden underwater movement of the sea-floor by using the time-history record of the water-wave elevation," Wave Motion, vol. 47, no. 3, pp. 146-155, 2010.

[11] M. Taylan, "The effect of nonlinear damping and restoring in ship rolling," Ocean Engineering, vol. 27, pp. 921-932, 2000.

FIGURE 6: The recovered (or identified) responses compared with the exact ones.

funded by the Ministry of Education, Science and Technology (Grant no. 2011-0010090). In addition, this research was also supported by Basic Science Research Program through the National Research Foundation of Korea (NRF) funded by the Ministry of Education, Science and Technology (Grant no. K20903002030-11E0100-04610). Finally, the author would like to extend his special thanks to the students, Miss. Hyeree Bae and Mr. Jinsoo Park in Naval Architecture and Ocean Engineering Department, Pusan National University for their help in the paper.

\section{References}

[1] M. Kulisiewicz, "A nonparametric method of identification of vibration damping in non-linear dynamic systems," International Journal of Solids and Structures, vol. 19, pp. 601-609, 1983.

[2] D. V. Iourtchenko and M. F. Dimentberg, "In-service Identification of non-linear damping from measured random vibration," Journal of Sound and Vibration, vol. 255, pp. 549-554, 2002. 


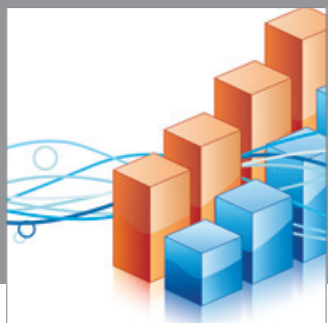

Advances in

Operations Research

mansans

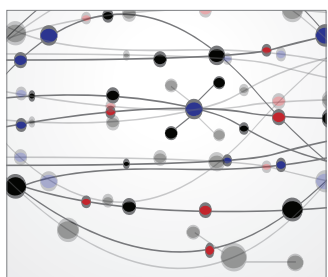

The Scientific World Journal
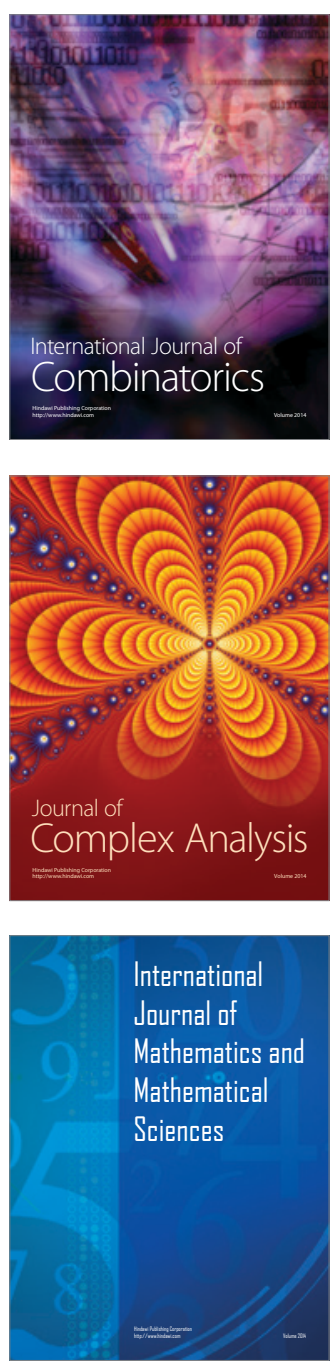
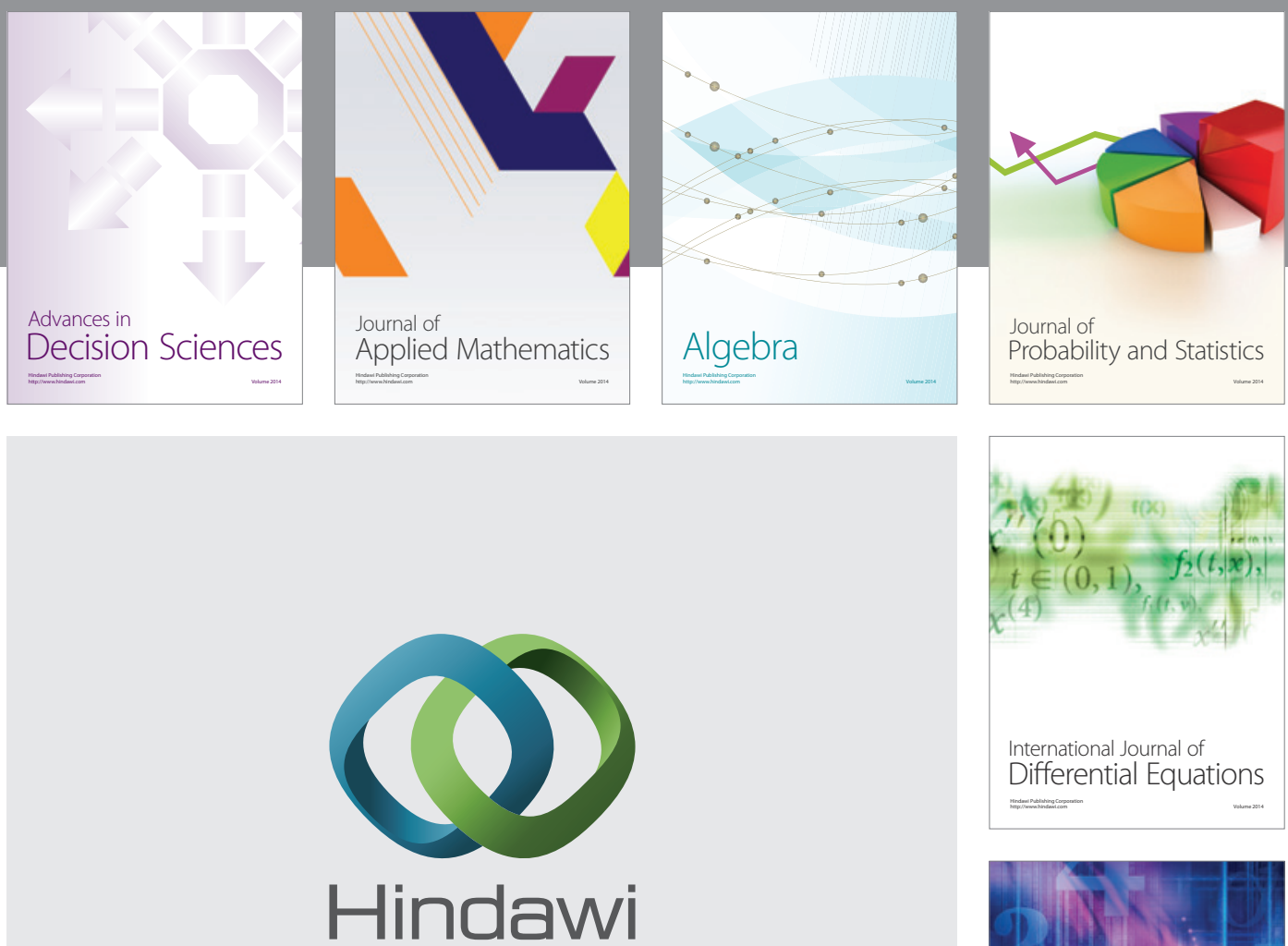

Submit your manuscripts at http://www.hindawi.com
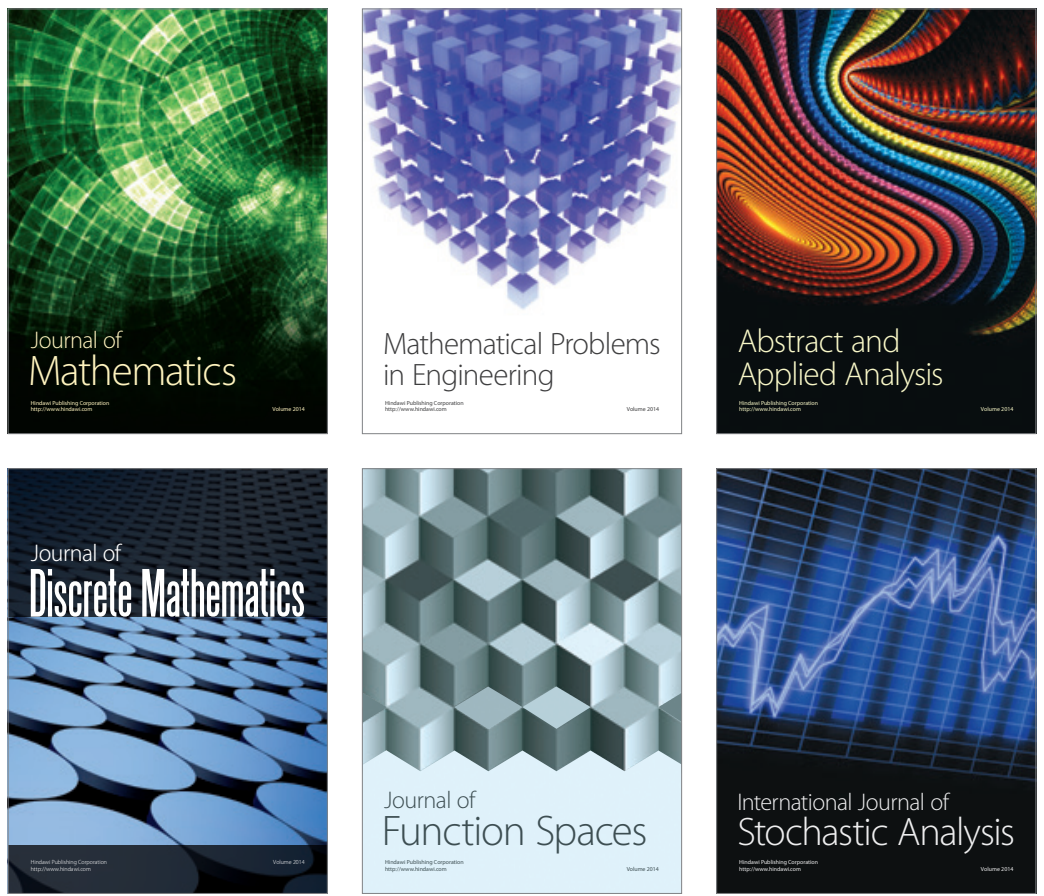

Journal of

Function Spaces

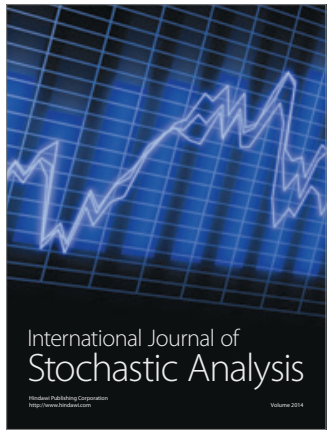

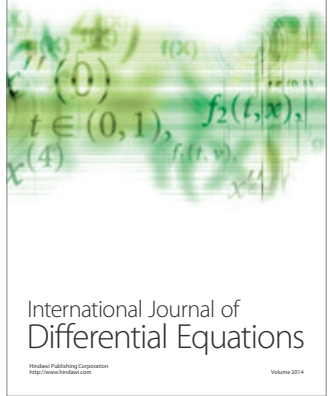
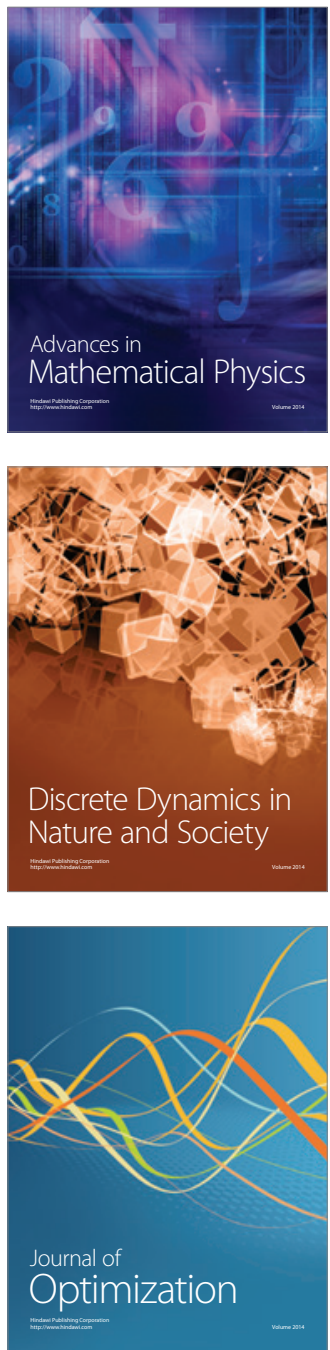